\title{
Activity of Muscles Surrounding Shoulder Joint during Push-Up Exercise according to the Change of Tilting Table Angle
}

\author{
Yong-Ho Cho, PT, PhD • Jin-Ho Choi, PT, $\mathrm{PhD}^{\dagger}$
}

Department of Physical Therapy, Daegu Haany University

Received: July 4, 2016 / Revised: July 16, 2016 / Accepted: August 5, 2016

(c) 2016 J Korean Soc Phys Med

\section{| Abstract |}

PURPOSE: This study was implemented to measure the muscle activity of muscles surrounding shoulder during push-up exercise according to the slope angle.

METHODS: This study has research subject consisting of 25 normal male adults without neurologic or musculoskeletal injury in shoulder part. EMG was used as a tool for measuring muscle activity and four muscles including upper trapezius (UT), middle trapezius (MT), Lower Trapezius (LT), and Serratus Anterior (SA) were chosen for measuring the muscle activity of muscles surrounding shoulder. Tilting table slope was set at $0^{\circ}, 30^{\circ}, 45^{\circ}$, and $60^{\circ}$ and push-up motion was performed three time for each case to use the average value. The measured value was used after generalization process to create \%RVC value where the measured value was standardized by using $0^{\circ}$ as a standard.

RESULTS: There was a difference of muscle activity according to the tilting table slope during push-up exercise. In UT, MT, and SA, there was no significant $0^{\circ}$ and $30^{\circ}, 45^{\circ}$ and $60^{\circ}$. But there was significant $30^{\circ}$ and $45^{\circ}$. LT was

†Corresponding Author : choipt88@gmail.com

This is an Open Access article distributed under the terms of the Creative Commons Attribution Non-Commercial License (http://creativecommons.org/licenses/by-nc/3.0) which permits unrestricted non-commercial use, distribution, and reproduction in any medium, provided the original work is properly cited. significant difference between $45^{\circ}$ and $60^{\circ}(\mathrm{p}<.05)$.

CONCLUSION: There are cases where push-up exercise should be selectively implemented due to shoulder problems. According to this results between $0^{\circ}$ and $30^{\circ}$, push up will be able to more effectively exercise. Applying proper angle of tilting table for push-up to patients who have difficulties in performing motions along with physical consumption can deliver effective and easy exercise program.

Key Words: Shoulder, EMG, Tilt Angle

\section{Introduction}

Working posture that requires sitting for long time induces repeated use of upper body and a posture where neck protrudes, which causes symptoms that induce neck and shoulder pain such as turtle neck syndrome. These symptoms are caused by imbalance of muscles surrounding neck and shoulder and they result in large inconvenience in daily life (Carter et al., 2001). Modernized lifestyle causes a number of problems. Lack of exercise, generalization of sedentary lifestyle, and increased use of computer and mobile phones induce habits of incorrect posture. This kind of habit consequently causes increase of pain in neck and shoulder part (Mok et al., 2004). To 
induce normal daily life by reducing the neck and shoulder pain, alignment and balance in neck and shoulder are important. Balanced development and proper coordination of muscles and tendons in shoulder complex are required for this (Carr and Shepherd, 2010). Shoulder has very wide range of movement and it is a joint that has the most complicated structure in body. The stability of joint itself is very weak and hence a lot of tendons and muscles play an important role in stability. Serratus anterior plays an important role in the stability of shoulder blade and it is important for overall shoulder stability (Watson and Schenkman, 1995). Stabilization of shoulder blade is critical in case of motor defects in shoulder joint and shoulder blade, impingement syndrome, and post-surgical management, for which muscles surrounding shoulder joint and shoulder blade are very important (Hintermeister et al., 1998).

Flexion of shoulder requires an exercise that accompanies internal and external rotation. However, for maximum flexion of shoulder joint, internal rotation of humerus should be followed, instead of external rotation. This is because internal rotation of humerus according to the action of tension takes place when bending upper arm over horizontal line as tension in tendon around shoulder joint acts (Mackinnon and Winter, 1993). When bending upper arm in external rotation condition on sagittal plane, complete flexion is possible when internal rotation of $45^{\circ}$ on horizontal plane takes place (McGill, 1991). This series of action are implemented by the interaction of tension that acts in shoulder joint tendon, structure of upper arm joint, and inactive tension of muscles in upper arm joint. However, these are results from the movement of rotation of humerus in shoulder movement. There are scarce data regarding specific measured values that indicate the difference of such movement (Richardson and Jull, 1995).

As such, advancement of society, sedentary lifestyle, and increased time of using computer have caused increase of shoulder diseases. There are a number of methods that treat the shoulder problems. There are diverse methods that manage shoulder disease, from expensive therapeutic exercise to easily implementable stretching (Jung et al., 2007). Among shoulder exercises that increase shoulder muscle strength, push-up is a representative exercise that people can easily perform at home without any special tool. Push-up is a method that can easily increase shoulder muscle strength without any tool. It is evaluated as a good exercise method for recovery after shoulder injury (Panjabi, 2003). Push up is good relative balance exercise between SA and UT (Ludewing et al., 2004). Problems of stress and posture resulting from work among office workers induce high frequency of musculoskeletal disease occurrence that accompanies pain in neck and shoulder. This trend is getting stronger following increasing amount of computer works and increasing number of office workers who work in this field. Hence, treatment and management of neck and shoulder disease is very important (Hall et al., 2004). This study measures muscle activity of muscles surrounding shoulder during push-up according to the slope angle. Specifically, this study measures muscle activity of upper trapezius (UT), middle trapezius (MT), lower trapezius (LT), and serratus anterior (SA) for the purpose of providing the most appropriate method during push-up exercise. The study is aimed to provide effective and efficient push-up exercise scheme through selective application of muscle strengthening method in case of weakened shoulder-surrounding muscles or rehabilitation program for shoulder.

\section{Method}

\section{Subjects}

This study has subject consisting of 25 normal male adults in their twenties without shoulder injury or pain. Among those without disease or injury in shoulder part, pain during daily activity, congenital deformity or disease, 
surgical damage or neurologic damage and disease were selected. All the subjects voluntarily agreed on participating in the experiment after listening to the explanation of the research purpose and method.

\section{Study tool and method}

\section{1) Experiment tool}

Surface electromyogram MP150 (Biopac system, USA) was used for the measurement of muscular contraction initiation time of shoulder muscles. Distance between active and inactive electrode was set at 20mm and TSD150B (composed of two stainless steels) was used for EMG signal measurement. Surface electromyogram signal was first stored in personal computer using software program Acqknowledge 3.8.2 (Biopac system, USA) and then analyzed. Data were collected where sampling rate was set at $1000 \mathrm{~Hz}$ to obtain EMG signal. Frequency acquisition domain was set at $30 \sim 500 \mathrm{~Hz}$ to obtain signal within normal range and $60 \mathrm{~Hz}$ notch filter was used for noise removal.

\section{2) Study method}

Four muscles including UT, MT, LT, and SA were measured for EMG signal in upper body muscles surrounding shoulder. Muscle belly of each muscle that is most prominently contracted in maximal muscular contraction in MMT posture was chosen for the site for attaching the active electrode. For accurate acquisition of EMG signal, skin resistance was reduced by first rubbing the skin of attaching site with thin sandpaper for three to four times to remove contaminants in strata cornea and cleansing with disinfecting alcohol. Moreover, for clear acquisition of the signal, skin tape was used for attaching the electrode to the skin. The reason of using skin tape was to remove mechanical noise caused by movement of research subject during the experiment and skin tape was used for fixating the electrode. Ground electrode was attached to $\mathrm{C} 7$ spinous process, which is a part that is not related to signal acquisition. As for the body slope for push-up, tilting table was used to first make the table horizontal with the ground with an angle $0^{\circ}$. The slope was raised to make the angle $30^{\circ}, 45^{\circ}$, and $60^{\circ}$ for the measurement. Break time of each trial was 1 minute. Push-up was performed three times in each slope and the average value was used for the measurement signal.

3) Treatment of muscle activity data

As for the data treatment method for the muscle activity data using EMG, test results from different subjects were compared through generalization process. In general, $\%$ Maximal Voluntary Contraction (\% MVC), \% Maximal Vonluntary Isometric Contraction ( $\%$ MVIC), and \% Reference Voluntary Contraction (\% RVC) are frequently used for standardization method. This study used \%RVC method. In the measurement according to the slope angle, push-up exercise on the ground $\left(0^{\circ}\right)$, which is most generally performed, can be used as a baseline for other slopes of tilting table. In case of $30^{\circ}, 45^{\circ}$, and $60^{\circ}$, the measured value was converted to \% RVC muscle activity measurement by using the measured value at push-up exercise on $0^{\circ}$ as a standard.

\section{Analysis method}

Measured data were analyzed by using SPSS and repeated measurement of ANOVA was used to compare the muscle activity according to the slope angle. A statistical significance level was set at .05.

\section{Results}

The study group's mean age, height, and weight were shown to be $23.3 \pm 1.4$ years, $172.4 \pm 3.9 \mathrm{~cm}$, and $71.1 \pm 8.1 \mathrm{~kg}$ respectively. All muscle activities decrease according to tilt angle increasing. In UT, MT, and SA, there was 
significant difference between $30^{\circ}$ and $45^{\circ}(\mathrm{p}<.05)$, but there was no significant difference between $0^{\circ}$ and $30^{\circ}$, $45^{\circ}$ and $60(\mathrm{p}>.05)$. And LT was significant difference between $45^{\circ}$ and $60^{\circ}(\mathrm{p}<.05)$, but there was no significant difference between $0^{\circ}$ and $30^{\circ}, 30^{\circ}$ and $45^{\circ}(\mathrm{p}>05$ ) (Table 1).

Table 1. Muscle activity on each tilt angle

(unit : \%RVC)

\begin{tabular}{llcc}
\hline & \multicolumn{1}{c}{$30^{\circ}$} & \multicolumn{1}{c}{$45^{\circ}$} & $60^{\circ}$ \\
\hline UT & $85.4 \pm 10.45^{*}$ & $70.07 \pm 29.92$ & $67.34 \pm 18.04$ \\
MT & $92.08 \pm 18.51^{*}$ & $76.94 \pm 26.81$ & $69.54 \pm 27.41$ \\
LT & $95.27 \pm 23.72$ & $76.75 \pm 25.96^{\dagger}$ & $48.14 \pm 26.98$ \\
SA & $93.88 \pm 20.25^{*}$ & $78.63 \pm 30.07$ & $77.24 \pm 21.16$ \\
\hline
\end{tabular}

UP : upper trazezius, MT : middle trapezius,

LT : lower trapezius, SA : serratus anterior

* significant difference between $30^{\circ}$ and $45^{\circ}$

${ }^{+}$significant difference between $45^{\circ}$ and $60^{\circ}$ $\mathrm{p}<.05$

\section{Discussions}

In this study, all muscles showed a difference in muscular activity, depending on the tilt angle. UT, MT, LT, and SA are the muscles that act in shoulder part and they are susceptible to discomfort and pain for those who have shoulder disease or pain. Study regarding the understanding about the mechanical movement and motor pattern of these muscles are important (Neumann, 2013). Injection is the method that can be most generally and easily applied when reducing shoulder pain, which is a disease that is frequently observed among modern people. Administrating steroid can bring effects such as increased range of motion of joint and pain reduction for patients with shoulder joint pain. For patients with chronic shoulder pain, administrating nerve block injection can bring similar effects including pain reduction. Such short-term effects require caution as pain management based on long-term use of medicines such as steroid has potential risk of side effects. Moreover, as there are limitations of fundamentally resolving mechanical problems in shoulder joint, diverse methods are required for shoulder management (Lee et al., 2007). Pain management for neck and shoulder through exercise is widely used recently. Stretching is one of the nonsurgical methods for reducing shoulder pain without medicine that people can easily use. In case of neck and shoulder pain resulting from work-related abnormal posture, regular and steady stretching can bring effects in neck and shoulder pain management (Kim and Lee, 2004). Recently, combined exercise is also receiving increasing amount of attention as an effective management scheme along with pilates as it showed effects of reducing shoulder and lower back pain. As such, pain management of shoulder is being applied by diverse methods (Yun et al., 2011). This study was implemented to find out the muscle activity of shoulder-surrounding muscles according to the different slope angle when performing push-up, which is a shoulder exercise that anyone can easily follow. According to the size of the tilting table slope, size of used power and pattern and shape of muscle will differ (Cho et al., 2015). Consequently, it is surmised that exercise effects will be also different. A lot of muscles will be used for muscular contraction when largest power is required. However, muscles that act more and those that act less will be different according to the tilting table slope. If this can be properly used, controlling the angle of tilting table can help patients who have difficulties in performing push-up on flat ground strengthen specific muscles.

There are studies about muscle activity according to shoulder variety condition. 4 exercises for shoulder rehabilitation program were shown variety muscle activities pattern (Moseley et al., 1992). Muscle contractions in Shoulder flexion, extension, abduction, internal / external rotation plays variety type, but muscle activities were not shown proportionately. Action of the muslces in the body is seen to be the veriaty according to the type of movement (Neumann, 2013). In this study, the activity of muscles appeared in various forms depending on the tilt level. 4 
muscles showed a significant decreasing of muscle activity according to the increasing tilt angle, but the patterns between angles were differences. In case of UT, MT, and $\mathrm{SA}$, there was no significant difference between $0^{\circ}$ and $30^{\circ}, 45^{\circ}$ and $60^{\circ}$. But there was a significant difference between $30^{\circ}$ and $45^{\circ}$. Muscle activities were acted different type. According this study result, muscle activities in $0^{\circ}$ and $30^{\circ}$ were not significant difference.

To sum up, muscle activity of muscles surrounding shoulder showed large difference according to the slope angle. If those with pain that are not able to perform the ordinary push-up exercise can receive similar effects from performing the push-up exercise in easier angle, push-up can be applied as therapeutic exercise program for many people. As there was no statistical difference of muscle activity between $0^{\circ}$ and $30^{\circ}$, push-up on $30^{\circ}$ can be implemented as an easier and more efficient exercise program for strengthening muscles than push-up on $0^{\circ}$.

\section{Conclusion}

This study was implemented to measure the muscle activity of 25 normal male people during push-up exercise in case of applying the slope angle of $0^{\circ}, 30^{\circ}, 45^{\circ}$, and $60^{\circ}$. It can be generally thought that size of muscle activity increases as slope angle decreases and size of muscle activity decreases as slope angle increases. But Statistical significance was not observed between $0^{\circ}$ and $30^{\circ}$. In case of patients who have difficulties in performing push-up exercise on flat ground due to shoulder injury or weakened shoulder part, push-up exercise using $30^{\circ}$ slope can be diversely used as an effective and active exercise program when exercise that strengthens specific muscles is required.

\section{Acknowledgements}

This research was supported by a grant from Daegu Haany University $\mathrm{Ky} \cdot \operatorname{lin}$ Foundation in 2014.

\section{References}

Carr JH, Shepherd RB. Neurological rehabilitation optimizing motor performance $\left(2^{\text {nd }}\right.$ ed) Scotland, Churchill Livingstone. 2010.

Carter ND, Kannus P, Khan KM. Exercise in the prevention of falls in older people: A systematic literature review examining the rationale and the evidence. Sports Med. 2001;31(6):427-38.

Cho YH, Kim SO, Choi JH. The differences of shoulder muscle activity onset time according to body tiliting angle in push-up exercise. J Korean Soc Phys Med. 2015;10(2):55-61.

Hall CD, Schubert MC, Herdman SJ. Prediction of fall risk reduction as measured by dynamic gait index in individuals with unilateral vestibular hypofunction. Otol Neurotol. 2004;25(5):746-51.

Hintermeister RA, Lange GW, Schultheis JM, et al. Electromyographic activity and applied load during shoulder rehabilitation exercises using elastic resistance. Am J Sports Med. 1998;26(2):210-20.

Jung YW, Bae SS, Jang WS. The effect of neural mobilization on the grip strength. J Korean Soc Phys Med. 2007;2(1):11-20.

Kim JK, Lee SJ. Effect of stretching exercise as work-related musculoskeletal pain of neck and shoulder. J Kor Alliance Health Phys Edu. 2004;43:655-62.

Lee EC, Kim HC, Jung DY, et al. Association between job-stress and VDT work, and musculoskeletal symptoms of neck and shoulder among white-collar workers. Korean J Occup and Environ Med. 2007;19(3):187-95.

Ludewig, PM, Hoff MS, Osowski EE, et al. Relative balance 
of serratus anterior and upper trapezius muscle activity during push-up exercises. Am J Sports Med. 2004;32(2):484-93.

MacKinnon CD, Winter DA. Control of whole body balance in the frontal plane during human walking. J Biomech. 1993;26(6):633-44.

McGill SM. Kinetic potential of the lumbar trunk musculature about three orthogonal orthopaedic axes in extreme postures. Spine. 1991;16(7):809-15.

Mok NW, Brauer SG, Hodges PW. Hip strategy for balance control in quiet standing is reduced in people with low back pain. Spine. 2004;29(6):107-12.

Moseley JB, Jobe FW, Pink M, et al. EMG analysis of the scapular muscles during a shoulder rehabilitation program. Am J Sports Med. 1992;20(2):128-34.
Neumann DA. Kinesiology of the musculoskeletal system: foundations for rehabilitation. St. Louis. Elsevier Health Sciences. 2013.

Panjabi MM. Clinical spinal instability and low back pain. J Electromyo Kinesiol. 2003;13(4):371-79.

Richardson CA, Jull GA. Muscle control-pain control. What exercises would you prescribe? Man Ther. 1995;1(1):2-10.

Watson CJ, Schenkman M. Physical therapy management of isolated serratus anterior muscle paralysis. Phys Ther. 1995;75(3):194-202.

Yun MJ, Byon JY, Kim HJ, et al. Effects of 12-week Pilates and Complex Exercises on Female Farm Workers of Cumulative Trauma Disorders. Health Sports Med. 2011;13(3):13-22. 